\title{
Rechallenge and maintenance therapy using cetuximab and chemotherapy administered to a patient with metastatic colorectal cancer
}

\author{
Jian Ma, Quan-liang Yang and Yang Ling
}

\begin{abstract}
Background: Cetuximab combined with chemotherapy is one of the first-line treatments of metastatic colorectal cancer. Although disease progression inevitably occurs, rechallenge and maintenance therapies using cetuximab-based regimens may be beneficial, particularly for patients with wild-type (WT) KRAS.

Case presentation: A 47-year-old female patient who underwent right hemicolectomy presented with an ulcerative adenocarcinoma (grade 2) revealed by histopathological analysis. The patient received three cycles of adjuvant chemotherapy, but disease recurred 15 months later. Cetuximab and a FOLFOX-4 regimen were administered, followed by surgery and adjuvant chemotherapy that was administered for approximately one year. Three years after completing adjuvant therapy, her serum carcinoembryonic antigen levels rapidly increased, and enhanced computed tomography showed widespread metastases. Rechallenge with cetuximab and the FOLFIRI regimen was then initiated, and after 12 cycles, lesions in the lung and liver shrank significantly, and serum CEA levels dramatically declined. Maintenance therapy with cetuximab and capecitabine was then administered for 10 months until the metastatic lesions in the lung and liver enlarged.
\end{abstract}

Conclusion: Rechallenge and maintenance therapy with cetuximab-based chemotherapy were relatively effective for managing a female patient with WT KRAS. Optimization of this strategy requires further in-depth investigations of more patients.

Keywords: Cetuximab, Metastatic colorectal cancer, Rechallenge, Maintenance therapy

\section{Background}

Colorectal cancer $(\mathrm{CRC})$ is one of the most common cancers worldwide. Approximately $60 \%$ of patients are diagnosed at a relatively late stage, and $71 \%$ with regionally distributed disease survive for 5 years. However, when disease has spread to distant organs, 5-year survival decreases to $13 \%$ [1]. One of the first-line options for treating patients with advanced CRC is the monoclonal antibody cetuximab that targets the epidermal growth factor receptor (EGFR) [2-4]. When combined with chemotherapy, cetuximab significantly improves progression-free survival and median overall survival of patients with wild-type (WT) KRAS

\footnotetext{
* Correspondence: lingyang2015@aliyun.com

Changzhou Cancer Hospital of Soochow University, Changzhou, Jiangsu

Province 213032, People's Republic of China
}

$[2,3]$. However, acquired resistance caused by a secondary mutation of KRAS occurs at a relatively high rate during cetuximab treatment [5]. For patients with WT KRAS, rechallenging initial responders with cetuximab-based regimens represents a promising strategy [6-8]. Here we present the case of a female patient who received three lines of therapy following her second surgery. Unfortunately, the disease metastasized to numerous organs, accompanied by a dramatic increase in serum carcinoembryonic antigen (CEA) levels before the rechallenge regimen commenced. The rechallenge and the subsequent maintenance therapies were highly beneficial for reducing the size of the lesions in lung and liver and reduced CEA levels. 


\section{Methods}

The drugs and antibodies used are as follows:

Cetuximab (Erbitux): Merck Serono Co., Ltd.

Capecitabine (Xeloda): Shanghai Roche Pharmaceuticals

Ltd.

Bevacizumab (Avastin): Shanghai Roche Pharmaceuticals

Ltd.

Oxaliplatin and irinotecan: Jiangsu Hengrui Medicine Co., Ltd

\section{Case presentation}

A 47-year-old woman who had undergone resection of the ascending colon on November 16, 2006, was admitted to our Department of Oncology with a diagnosis after surgery of ulcerative adenocarcinoma (grade 2) of the ascending colon. The tumor penetrated to the surface of the visceral peritoneum, and metastases were detected in five regional lymph nodes (pT4aN2aM0). In accordance with National Comprehensive Cancer Network Guidelines in Oncology [9], we initiated adjuvant therapy using the XELOX regimen comprising oxaliplatin $\left(130 \mathrm{mg} / \mathrm{m}^{2}\right.$, Day 1$)$ and capecitabine $\left(1000 \mathrm{mg} / \mathrm{m}^{2}\right.$, p.o bid Days 1-14), 21 days per cycle. After three cycles, the patient refused to continue therapy due to the onset of leukopenia (grade 3), in accordance with the guidelines of the NCI Common Terminology Criteria for Adverse Events (CTCAE v 4.0) [10].

On February 20, 2008, a routine examination revealed that her serum carcinoembryonic antigen (CEA) concentration increased to $24.41 \mathrm{ng} / \mathrm{ml}$ without symptoms. The results of positron-emission tomography with computed tomography (PET-CT) showed increased metabolic activities of the right adnexa mass and hepatic hilar region node. At that time, her KRAS status was unknown and such testing was not a mandatory requirement of the China Food and Drug Administration (CFDA). On March 1, 2008, the patient started to receive cetuximab and the FOLFOX4 regimen (Day 1: 5 -FU $400 \mathrm{mg} / \mathrm{m}^{2}$ bolus injection; LV $200 \mathrm{mg} / \mathrm{m}^{2}$; $22 \mathrm{~h}$ continuous infusion with 5 -FU $600 \mathrm{mg} / \mathrm{m}^{2}$, and oxaliplatin $85 \mathrm{mg} / \mathrm{m}^{2}$; Day 2: the same regimen without oxaliplatin, each 14-day cycle). Cetuximab was administered (initial dose of $400 \mathrm{mg} / \mathrm{m}^{2}$ ) followed by $250 \mathrm{mg} / \mathrm{m}^{2}$ per week. Four cycles later, abdominal enhanced CT demonstrated a partial response (PR) of the metastases according to the Response Evaluation Criteria in Solid Tumors (RECIST 1.0), and the patient underwent right adnexectomy and partial gastrointestinal ligament resection. One month after surgery, two additional cycles of FOLFOX4 regimen (see above) were administered. However, we changed to the FOLFIRI regimen for two cycles because of neuropathy (sensory, grade 2) caused by oxaliplatin and the patient's refusal of FOLFOX4. However, because the patient suffered from the severe chest tightness and fatigue (grade 2), we discontinued the FOLFIRI regimen and started oral administration of capecitabine (1000 mg/ $\mathrm{m}^{2}$, p.o bid, Days 1-14 every three weeks for 10 months).

During the next three years, routine examinations did not detect recurrence, although the patient experienced pain around the anus. In November 2012, her serum CEA increased to $>1,000 \mathrm{ng} / \mathrm{ml}$. CT of the chest and abdomen revealed widely distributed metastases, including multiple involved lymph nodes in the mediastinum, pelvic cavity, and behind the peritoneum, liver, and both lungs (Fig. 1a). Molecular analysis identified WT KRAS. From November 2012, the patient was rechallenged weekly with cetuximab (400 mg/m $\mathrm{m}^{2}$ initially and $250 \mathrm{mg} / \mathrm{m}^{2}$ thereafter) and FOLFIRI (Day 1: Irinotecan $180 \mathrm{mg} / \mathrm{m}^{2} ; 5-\mathrm{FU} 400 \mathrm{mg} / \mathrm{m}^{2}$ bolus injection; 5 -FU $600 \mathrm{mg} / \mathrm{m}^{2}, 22 \mathrm{~h}$ continuous infusion; LV $200 \mathrm{mg} / \mathrm{m}^{2}$; Day 2:the same regimen without irinotecan; each 14-day cycle). After two cycles of treatment, the pain around the anus was relieved, although the CEA concentration did not decline ( $>1000 \mathrm{ng} / \mathrm{ml}$ ) (Fig. 2). Four cycles later, chest and abdominal enhanced CT demonstrated that all the lesions shrank significantly (PR, according to RECIST 1.1) (Fig. 1b), and the CEA concentration was significantly reduced to $92.5 \mathrm{ng} / \mathrm{ml}$ (Fig. 2). Eight and twelve cycles later, chest and abdominal enhanced CT showed that all lesions shrank further and the CEA concentrations were reduced to $17.89 \mathrm{ng} / \mathrm{ml}$ and $6.6 \mathrm{ng} / \mathrm{ml}$, respectively (Figs. 1c, d and Fig 2). During treatment, rash, eyelash trichomegaly and myelosuppression (grade 2) occurred.

We then changed the treatment strategy to capecitabine single-agent chemotherapy $\left(750 \mathrm{mg} / \mathrm{m}^{2}\right.$ p.o bid, Days $1-$ 21 , each 28 -day cycle) combined with cetuximab (250 mg/ $\mathrm{m}^{2}$ weekly) as maintenance therapy, which led to further shrinkage of the lesions after two cycles (Fig. 1e). We continued maintenance therapy for approximately 10 months (April 2014), when the lesions in the lung and the liver progressed (PD). Peripheral neuropathy was improved at that time, and the patient had a strong desire to survive; she therefore accepted our advice and tried the FOLFOX4 regimen again, risking its potentially severe side effects. We switched to bevacizumab $(5 \mathrm{mg} / \mathrm{kg}$, once every two weeks) plus the FOLFOX4 regimen for four cycles. CT showed stable disease; however, she refused intravenous chemotherapy because of intolerance. We therefore replaced FOLFOX4 with capecitabine. Progression-free survival lasted from April through November, and she was then administered regorafenib for nearly two months, which was terminated because of financial difficulties. We then administered cetuximab plus capecitabine for approximately three months, followed by the best available supportive care until she died on August 29, 2015.

\section{Discussion}

The cetuximab-based regimen is one of the first-line options for treating patients with advanced colorectal 


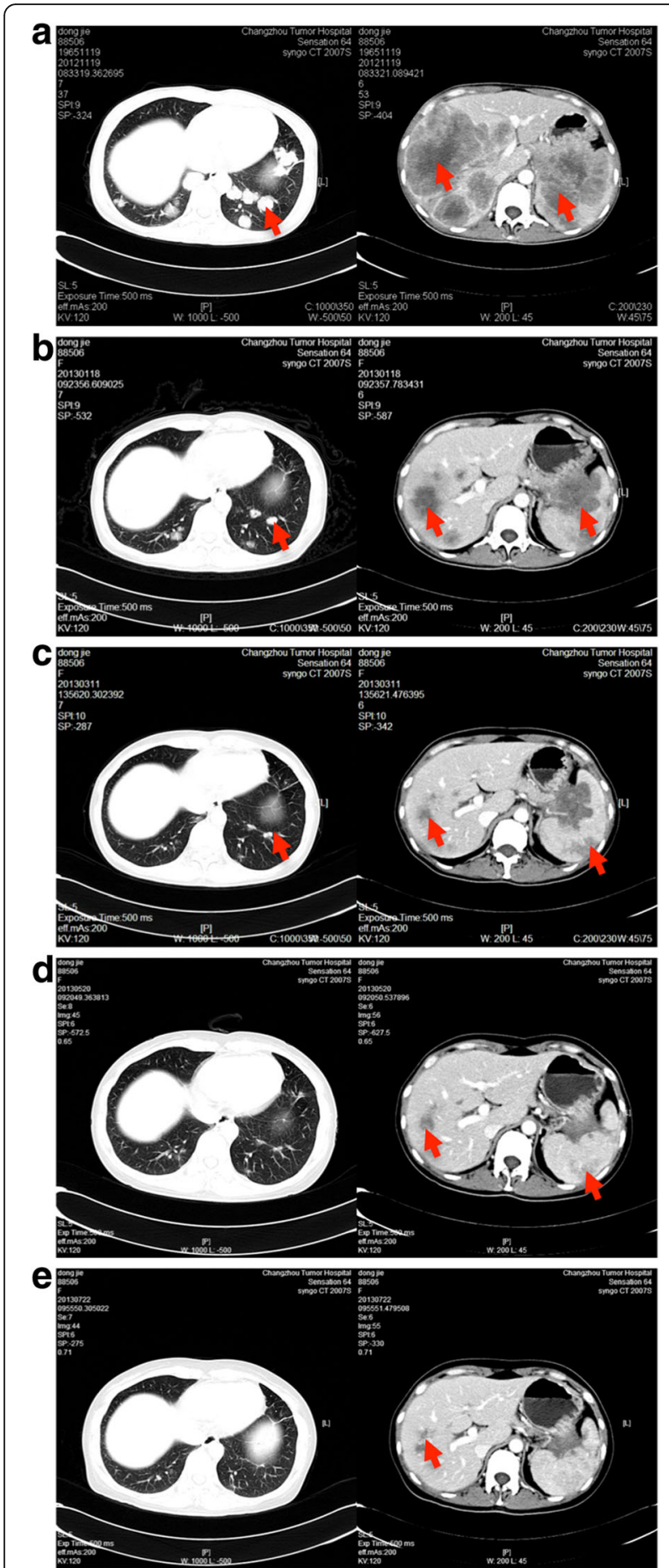

Fig. $1 C T$ results showing the metastatic lesions in the liver, lung and kidney before (a), four (b), eight (c), and twelve cycles (d) after cetuximab and FOLFIRI rechallenge, and two cycles (e) after cetuximab and capecitabine maintenance therapy. Arrows indicate the lesions

cancer. Cetuximab is a chimeric IgG1 monoclonal antibody against EGFR that inhibits the ligand-induced EGFR signaling pathway through binding to the extracellular

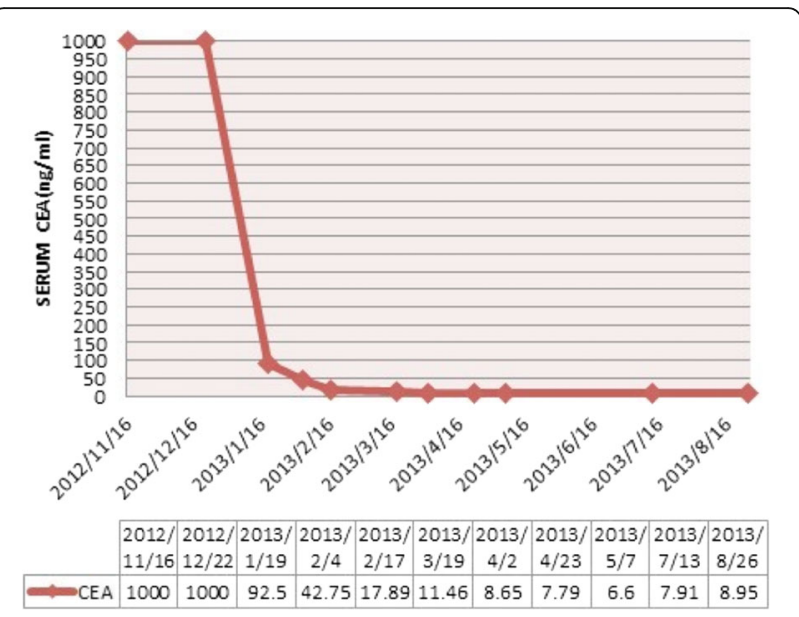

Fig. 2 CEA levels of the patient

domain of EGFR, which inactivates EGFR and its downstream factors [11].

Clinical trials demonstrate the benefit of cetuximab in combination with chemotherapy for treating patients with metastatic colorectal cancer (mCRC) $[12,13]$. However, because $\mathrm{mCRC}$ is heterogeneous, cetuximab-based regimens only benefit patients with WT KRAS, a downstream effector of the EGFR signaling pathway [14]. Mutational activation of $K R A S$ can confer primary resistance to EGFR-targeted therapies, including cetuximab [12-14]. Nevertheless, for initial responders who harbor WT KRAS or silent KRAS mutations, rechallenge with cetuximab may be further clinically beneficial if patients do not respond to a new line chemotherapy and therefore receive other therapies [8].

Several clinical trials tested this hypothesis and demonstrated favorable efficacies of cetuximab-based rechallenge regimens $[8,15]$. Notably, rebiopsy may be required when rechallenge is considered for these patients, because secondary KRAS mutations may confer acquired resistance to EGFR-targeted therapy [5]. Here we report a female patient who was administered three lines of therapy after the second surgery without an activating KRAS mutation. Rechallenge with cetuximab caused a beneficial and dramatic shrinkage of metastatic lesions in the lung and liver as well as a significant reduction in CEA levels, further supporting the expectation of a promising outcome of a rechallenge using cetuximab.

Maintenance therapy is an important approach for improving the outcomes of patients with cancer who receive certain lines of chemotherapy to prolong the duration of therapy to control long-term cancer growth. The phase III CAIRO3 trial explored the efficacy of maintenance therapy with capecitabine plus bevacizumab, compared with the observation group in patients who achieve at least stable disease after six cycles (18 weeks) of induction therapy with capecitabine, oxaliplatin and bevacizumab 
(CAPOX-B) [16]. The conclusion drawn from this trial was that maintenance therapy significantly delayed tumor progression and did not compromise a patient's quality of life. However, the effect of cetuximab-based maintenance therapy has not been conclusively investigated.

According to the findings of the CAIRO3 trial, we tested here the efficacy of maintenance therapy using cetuximab and capecitabine and found further shrinkage of metastatic lesions after two cycles. The disease progressed after 10 months of maintenance therapy, suggesting that in addition to rechallenge, maintenance treatment with a cetuximab-based regimen may potentially benefit the patient. Further validation and optimization of this strategy with more patients should be conducted.

Optimizing the sequence of administration of cetuximab and bevacizumab may influence overall survival, because the CRYSTALY and FIRE-3 trials found that early tumor shrinkage was more likely to occur after cetuximab treatment that improves the R0 removal rate of the tumor [4, 12]. Further, single-agent maintenance therapy using cetuximab should be evaluated to determine the efficacy of cetuximab in patients treated with FOLFIRI, as reported in the MACRO-II trial, which found that cetuximab alone achieves similar benefits with fewer side-effects compared with mFOLFOX plus cetuximab [17]. Moreover, it will likely be informative to evaluate the tumor response to reintroduction of FOLFIRI plus cetuximab when maintenance therapy fails, as indicated by the reintroduction of CAPOX-B in the CAIRO-3 trial [16].

A recent complete exome sequencing study identified mutations in ERBB2, EGFR, FGFR1, PDGFRA and MAP2K1 as potential drivers of resistance to EGFRtargeted therapies [18]. Therefore, it may be useful to determine the efficacies of approaches that target these genes in combination with cetuximab for rechallenge and maintenance treatment.

The patient died more than one year before the preparation of this manuscript. The subsequent emergence of new treatments has provided more options for patients with similar characteristics to the present patient. For example, trifluridine-tipiracil (TAS-102) is approved by the FDA, and in the treatment of refractory colorectal cancer, the median overall survival improved from 5.3 months with placebo to 7.1 months with TAS-102 [19]. Pembrolizumab is active in patients with mismatch repair-deficient colorectal cancer (response rate, 40\%; 95\% confidence interval, $12-74 \%$ ) [20]. These regimens may achieve better outcomes.

\section{Conclusion}

Rechallenge with cetuximab and FOLFIRI may be effective for treating patients with $\mathrm{mCRC}$, and subsequent maintenance therapy with cetuximab and capecitabine may provide an option for selected patients.

\section{Abbreviations}

CEA: Carcinoembryonic antigen; CFDA: China Food and drug administration; CT: Computed tomography; EGFR: Epidermal growth factor receptor;

FOLFIRI: Folinic acid, fluorouracil and irinotecan; mCRC: Metastatic colorectal cancer; PET-CT: Positron-emission tomography with computed tomography; WT: Wild-type

\section{Acknowledgements}

We thank the staff of the Department of Anatomic Pathology of Changzhou Cancer Hospital of Soochow University for performing the molecular analysis.

\section{Funding}

This study was supported by Changzhou Sci\&Tech Program, China (Grant No.: CE20155043, CJ20160049)

\section{Availability of data and materials}

The datasets used and/or analyzed during the current study are available from the corresponding author on reasonable request.

\section{Authors' contributions}

JM, QY and YL designed the study; JM and QY acquired the data; JM and YL analyzed and interpreted the data and drafted the manuscript. All authors

have read and approved the manuscript.

\section{Competing interests}

The authors declare that they have no competing interests.

\section{Consent for publication}

Written informed consent was obtained from the patient for publication of this case report and any accompanying images. A copy of the written consent is available for review by the Editor of this journal.

Ethics approval and consent to participate

This study was approved by the Ethical Committee of Changzhou Cancer Hospital of Soochow University.

Received: 20 November 2015 Accepted: 10 February 2017

Published online: 14 February 2017

\section{References}

1. Cancer Facts \& Figures 2015. https://old.cancer.org/acs/groups/content/ @editorial/documents/document/acspc-044552.pdf.

2. Hurwitz H, Fehrenbacher L, Novotny W, Cartwright T, Hainsworth J, Heim W Berlin J, Baron A, Griffing S, Holmgren E, et al. Bevacizumab plus irinotecan, fluorouracil, and leucovorin for metastatic colorectal cancer. N Engl J Med. 2004;350(23):2335-42.

3. Cunningham $D$, Humblet $Y$, Siena $S$, Khayat $D$, Bleiberg $H$, Santoro A, Bets $D$,

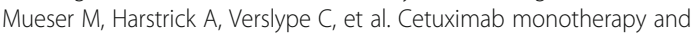
cetuximab plus irinotecan in irinotecan-refractory metastatic colorectal cancer. N Engl J Med. 2004;351(4):337-45.

4. Van Cutsem E, Köhne CH, Hitre E, Zaluski J, Chang Chien CR, Makhson A, D'Haens G, Pintér T, Lim R, Bodoky G, et al. Cetuximab and chemotherapy as initial treatment for metastatic colorectal cancer. N Engl J Med. 2009; 360(14):1408-17

5. Misale S, Yaeger R, Hobor S, Scala E, Janakiraman M, Liska D, Valtorta E, Schiavo R, Buscarino M, Siravegna G, et al. Emergence of KRAS mutations and acquired resistance to anti-EGFR therapy in colorectal cancer. Nature. 2012:486(7404):532-6.

6. Gattenlöhner S, Etschmann B, Kunzmann V, Thalheimer A, Hack M, Kleber G, Einsele $\mathrm{H}$, Germer C, Müller-Hermelink HK. Concordance of KRAS/BRAF mutation status in metastatic colorectal cancer before and after anti-EGFR therapy. J Oncol. 2009;2009:831626.

7. Zhu D, Keohavong P, Finkelstein SD, Swalsky P, Bakker A, Weissfeld J, Srivastava S, Whiteside TL. K-ras gene mutations in normal colorectal tissues from K-ras mutation-positive colorectal cancer patients. Cancer Res. 1997; 57(12):2485-92.

8. Santini D, Vincenzi B, Addeo R, Garufi C, Masi G, Scartozzi M, Mancuso A Frezza AM, Venditti O, Imperatori $M$, et al. Cetuximab rechallenge in 
metastatic colorectal cancer patients: how to come away from acquired resistance? Annals of oncology : official journal of the European Society for Medical Oncology/ESMO. 2012;23(9):2313-8.

9. New NCCN Guidelines Include Evidence Blocks to Illustrate Value in Breast Colon, Kidney, and Rectal Cancers. J Natl Compr Canc Netw 2016, 14(3): xxxiv-xxxv.

10. Health UDo, Services $\mathrm{H}$ : Common terminology criteria for adverse events (CTCAE) version 4.0. National Institutes of Health, National Cancer Institute 2009, 4(03).

11. Goldstein NI, Prewett M, Zuklys K, Rockwell P, Mendelsohn J. Biological efficacy of a chimeric antibody to the epidermal growth factor receptor in a human tumor xenograft model. Clin Cancer Res. 1995;1(11):1311-8.

12. Heinemann $V$, von Weikersthal LF, Decker T, Kiani A, Vehling-Kaiser U, Al-Batran SE, Heintges T, Lerchenmüller C, Kahl C, Seipelt G, et al. FOLFIRI plus cetuximab versus FOLFIRI plus bevacizumab as first-line treatment for patients with metastatic colorectal cancer (FIRE-3): a randomised, open-label, phase 3 trial. Lancet Oncol. 2014;15(10):1065-75.

13. Wasan H, Meade AM, Adams R, Wilson R, Pugh C, Fisher D, Sydes B, Madi A, Sizer B, Lowdell C, et al. Intermittent chemotherapy plus either intermittent or continuous cetuximab for first-line treatment of patients with KRAS wild-type advanced colorectal cancer (COIN-B): a randomised phase 2 trial. Lancet Oncol. 2014;15(6):631-9.

14. Markman B, Javier Ramos F, Capdevila J, Tabernero J. EGFR and KRAS in colorectal cancer. Adv Clin Chem. 2010;51:71-119.

15. Liu X, George GC, Tsimberidou AM, Naing A, Wheler JJ, Kopetz S, Fu S, Piha-Paul SA, Eng C, Falchook GS, et al. Retreatment with anti-EGFR based therapies in metastatic colorectal cancer: impact of intervening time interval and prior anti-EGFR response. BMC Cancer. 2015;15:713.

16. Simkens LH, van Tinteren H, May A, ten Tije AJ, Creemers GJ, Loosveld OJ, de Jongh FE, Erdkamp FL, Erjavec Z, van der Torren AM, et al. Maintenance treatment with capecitabine and bevacizumab in metastatic colorecta cancer (CAIRO3): a phase 3 randomised controlled trial of the Dutch colorectal cancer group. Lancet. 2015:385(9980):1843-52.

17. Alfonso PG, Benavides M, Ruiz AS, Guillen-Ponce C, Safont M, Alcaide J, Gómez A, Lopez R, Manzano J, Urena MM. 499ophase ii study of first-line mfolfox plus cetuximab (c) for 8 cycles followed by mfolfox plus $\mathrm{c}$ or single agent $(\mathrm{s} / \mathrm{a}) \mathrm{c}$ as maintenance therapy in patients (p) with metastatic colorectal cancer (mcrc): the macro-2 trial (spanish cooperative group for the treatment of digestive tumors [ttd]). Annals of Oncology. 2014;25(4):iv168.

18. Bertotti A, Papp E, Jones S, Adleff V, Anagnostou V, Lupo B, Sausen M, Phallen J, Hruban CA, Tokheim C, et al. The genomic landscape of response to EGFR blockade in colorectal cancer. Nature. 2015;526(7572):263-7.

19. Mayer RJ, Van Cutsem E, Falcone A, Yoshino T, Garcia-Carbonero R, Mizunuma N, Yamazaki K, Shimada Y, Tabernero J, Komatsu Y, et al. Randomized trial of TAS-102 for refractory metastatic colorectal cancer. N Engl J Med. 2015:372(20):1909-19.

20. Le DT, Uram JN, Wang H, Bartlett BR, Kemberling $H$, Eyring AD, Skora AD, Luber BS, Azad NS, Laheru D, et al. PD-1 blockade in tumors with mismatch-repair deficiency. N Engl J Med. 2015;372(26):2509-20.

\section{Submit your next manuscript to BioMed Central and we will help you at every step:}

- We accept pre-submission inquiries

- Our selector tool helps you to find the most relevant journal

- We provide round the clock customer support

- Convenient online submission

- Thorough peer review

- Inclusion in PubMed and all major indexing services

- Maximum visibility for your research

Submit your manuscript at www.biomedcentral.com/submit 